\title{
Gestión de capital de trabajo y su efecto en la rentabilidad para el grupo de empresas del sector manufacturero Ecuatoriano
}

\section{Management of working capital and its effect on profitability for the group of companies in the Ecuadorian manufacturing sector}

DOI: $10.46932 / \mathrm{sjjdv2n2-075}$

Received in: March 1st, 2021

Accepted in: May 30th, 2021

\section{Cesar Augusto Valladares Guamán}

Magister en Finanzas con mención en Gestión Financiera Internacional, por la Pontificia Universidad Católica del Ecuador, Sede Santo Domingo, Ecuador

Institución: Instituto Superior Tecnológico Tsáchila

Dirección: Avenida los Anturios y Calle B, Santo Domingo de los Tsáchilas, Ecuador

E-mail: cesarvalladares@tsachila.edu.ec, cavalladaresg@pucesd.edu.ec

\section{Karen Valeria Sánchez Jiménez}

Magister en Finanzas con mención en Gestión Financiera Internacional, por la Pontificia Universidad

Católica del Ecuador, Sede Santo Domingo, Ecuador

Institución: Instituto Tecnológico Superior Japón

Dirección: Av. Galápagos 505 y Calle Cuenca (frente a Solca)

E-mail: ksanchez@itsjapon.edu.ec,kvsanchezj@pucesd.edu.ec

\section{Mikel Ugando Peñate}

Doctor en Economía, por la Universidad de Santiago de Compostela, España

DEA en Programa Doctorado Convergencia Internacional en el Ámbito Financiero y Contable, por la

Universidad de Vigo, España

Institución: Pontificia Universidad Católica del Ecuador, Sede Santo Domingo, Ecuador

Dirección: Vía Chone Km 2, Santo Domingo, Ecuador, CP: 230203

E-mail: upm@pucesd.edu.ec, ugando75@gmail.com

\section{Ángel Ramon Sabando García}

Magister en Estadística Aplicada, por la Universidad técnica de Manabí, Ecuador Magister en Ingeniería Agrícola, por Universidad Estatal del Sur de Manabí, Ecuador Institución: Pontificia Universidad Católica del Ecuador, Sede Santo Domingo, Ecuador

Dirección: Vía Chone Km 2, Santo Domingo, Ecuador, CP: 230203

E-mail: sgar@pucesd.edu.ec

\section{Antonio Villalón Peñate}

Magister en Finanzas con mención en Gestión Financiera Internacional, por la Pontificia Universidad Católica del Ecuador, Sede Santo Domingo, Ecuador

Dirección: Vía Chone Km 2, Santo Domingo, Ecuador, CP: 230203

E-mail: antoniovillalon1987@gmail.com ${ }_{2}$ avillalonp@pucesd.edu.ec,

\section{RESUMEN}

La industria manufacturera en el Ecuador desempeña un papel preponderante en el ámbito de la economía del país, este sector favorece a la generación de ingresos, empleos y propende a la concreción de oportunidades de desarrollo. El presente estudio tiene como propósito analizar el efecto de la gestión de 
capital de trabajo en la rentabilidad de las empresas del sector manufacturero. La investigación se realizó con base a un enfoque cuantitativo de tipo descriptivo, longitudinal y correlacional. Para la recolección de información se aplicó análisis de contenidos, por cuanto se seleccionaron datos respecto a los indicadores de gestión y rentabilidad de las empresas correspondientes a los subsectores del área de manufactura, haciendo referencia a una indagación de cinco años al cierre del periodo 2018 según la información emitida por la Superintendencia de Compañías. Posteriormente se estableció la inducción del coeficiente de correlación y análisis multivalente con base al modelo de regresión múltiple para determinar el nivel de relación de diversos indicadores de capital de trabajo en referencia a la rentabilidad del activo. Como principales resultados se identifica que los modelos globales son significativos para los periodos 2014-2017, implicando que los indicadores de gestión de capital de trabajo explican las fluctuaciones de rentabilidad del activo, sin embargo, en el año 2018 el modelo no es significativo. La variable independiente que mayor incidencia genera en la rentabilidad es la rotación de ventas, puesto que su significancia se replica en tres años consecutivos y las pruebas de bondad de ajuste son significativas y los coeficientes diferentes de cero. Para el análisis matemático, estadístico y gráfico se utilizó las aplicaciones informáticas Microsoft® Excel (2019), y SPSS versión 21.

Palabras clave: gestión, capital de trabajo, rentabilidad, correlación, manufactura

\begin{abstract}
The manufacturing industry in Ecuador plays a leading role in the country's economy, favoring the generation of income, employment and the realization of development opportunities. The purpose of this study is to analyze the impact of working capital management on the profitability of enterprises in the manufacturing sector. The research was conducted based on a quantitative approach of descriptive, longitudinal and correlational type. Content analysis was applied to the collection of information, since data were selected for the management and profitability indicators of enterprises in the manufacturing subsectors, referring to a five-year investigation at the end of the 2018 period according to information issued by the Superintendence of Companies. Subsequently, the induction of the correlation coefficient and multivalent analysis were established based on the multiple regression model to determine the level of relation of various working capital indicators in reference to the profitability of the asset. As main results it is identified that the global models are significant for the periods $2014-2017$, implying that the indicators of management of working capital explain the fluctuations of profitability of the asset, however in the year 2018 the model is not significant. The independent variable that generates the greatest impact on profitability is sales turnover, since its significance is replicated in three consecutive years and the goodness-of-fit tests are significant and the coefficients are different from zero. For the mathematical, statistical and graphic analysis, the computer applications Microsoft@ Excel (2019), and SPSS version 21 were used.
\end{abstract}

Keywords: management, working capital, profitability, correlation, manufacturing

\title{
1 INTRODUCCIÓN
}

El estudio del capital de trabajo ha sido abordado desde diferentes contextos tomando en consideración la relevancia de las actividades operacionales de las empresas o sectores empresariales estudiados. Miller (2014) en su análisis de la administración del capital de trabajo y rentabilidad del sector textil en un sector de Cúcuta, establece que las variaciones del Capital de Trabajo Neto Operativo (KTNO) incidieron en un 89,4\% en las fluctuaciones de la Rentabilidad del Activo Neto (RAN) y en un 98,1\% las 
variaciones de la Rentabilidad del Patrimonio (ROE). Las Cuentas por Cobrar (CxC) representaron el $96,67 \%$ de las variaciones del ROE.

Rotstein, et. al. (2004), efectuaron una investigación de la gestión del capital de trabajo en Pymes de capital local, en Bahía Blanca, Argentina. El estudio estableció que la ineficiencia en la gestión de capital de trabajo desencadena una baja rentabilidad de las empresas de este sector. En este contexto dicha perspectiva de ineficiencia direcciona a buscar fuentes de financiamiento externo, con altos costos financieros. Angulo y Berrio (2014), concretaron un estudio direccionado a las pequeñas y medianas empresas colombianas de comercio textil, analizando diversas estrategias de inversión en capital de trabajo, desde un contexto netamente financiero. Los resultados evidenciaron que la gran parte de este sector empresarial dispone de un alto nivel de ciclo de conversión de efectivo, lo cual evidencia la inadecuada gestión del capital de trabajo.

Vera, Melgarejo y Mora (2013), desarrollaron una investigación a 4168 Pymes colombianas, en los periodos 2004 a 2009. El objetivo fue establecer y relacionar los indicadores y las fuentes de financiación. Con base a dicho planteamiento la mayoría de empresas estudiadas financian sus actividades con recursos propios y pasivos de corto plazo, pudiendo incidir de esta manera desfavorablemente en los resultados de indicadores de gestión. Esta tendencia limita la obtención de recursos para generar una operación eficiente y lograr un crecimiento sostenido.

Cuenca (2018), desarrolla un estudio a una muestra de 312 empresas constructoras para identificar la relación del capital de trabajo en ese sector empresarial ecuatoriano, concluyendo que el periodo de rotación del inventario (PRI), periodo medio de cobranzas (PMC), periodo medio de pagos (PMP), prueba ácida (PA) y el endeudamiento del activo (LEV) tienen relación negativa significativa con el ROA. Estos resultados determinan la tendencia del comportamiento de los indicadores estudiados, puesto que en la practica a nivel empresarial una disminución de los periodos de cobranza o minimización del endeudamiento del activo podria suponer un incremento de la rentabilidad.

En los últimos años la industria manufacturera ecuatoriana se ha visto afectada por los declives económicos, tal es el caso que en la actualidad según registros emitidos por el Servicio de Rentas Internas (SRI) este sector a disminuido sus ventas en un $27,3 \%$, y la perspectiva furura no es provisoria. El sector manufacturero está estructurado por 24 subsectores según la Clasificación Industrial de Clasificación Uniforme (CIIU) Rev. 4, y durante el periodo 2014-2018 se contabilizaron 7058 industrias. (Superintendencia de Compañías, 2020).

La industria manufacturera representa un rol sumamente relevante en la producción ecuatoriana, Horma \& Osorio (2009). En un contexto de análisis general se estipula que las ventas y salarios de estas 
empresas constituyen aproximadamente el $20 \%$ del sector societario, de la misma forma evidencian altos niveles de rentabilidad (ROA y ROE). (Superintendencia de Compañías, 2018).

Con base a este contexto se define como problemática que, los representantes de las compañías del sector manufacturero ecuatoriano deben generar condiciones óptimas de gestión de capital de trabajo, por cuanto es fundamental conocer el comportamiento de diversos indicadores frente a la fluctuación de la rentabilidad, información que puede generar lineamientos estratégicos para la toma de decisiones, sin embargo, no se han establecido estudios específicos que permitan responder a la siguiente interrogante: ¿Cómo la gestión de capital de trabajo afecta la rentabilidad de las empresas del sector manufacturero ecuatoriano?. Definiéndose como objetivo general de la investigación: Analizar el efecto de la gestión de capital de trabajo en la rentabilidad de las empresas del sector manufacturero ecuatoriano a través de la aplicación de modelos estadísticos.

La presente investigación se encuentra alineado al Plan Nacional de Desarrollo Económico Toda una Vida 2017 - 2021, específicamente en el eje $\mathrm{N}^{\mathrm{o}} 2$ objetivo $\mathrm{N}^{\mathrm{0}}$ 5, que establece: "Impulsar la productividad y competitividad para el crecimiento económico sostenible de manera redistributiva y solidaria el cual prioriza la consolidación de la sostenibilidad del sistema económico social y solidario, y fortalecimiento de la dolarización" (Senplades, 2017, p. 2017).

\section{REVISIÓN DE LITERATURA}

\subsection{CAPITAL DE TRABAJO NETO EN EL CONTEXTO EMPRESARIAL}

Desde una perspectiva elemental Gitman \& Zutter (2012) consideran que el capital de trabajo neto o fondo de maniobra representa "La diferencia entre los activos corrientes de la empresa y sus pasivos corrientes". Desde un contexto financiero según Rizzo (2007) y Abreu \& Morales 2009 "El capital de trabajo neto es la cantidad de dinero que la empresa necesita para mantener el giro habitual del negocio". Este concepto abarca una apreciación más amplia que determina la importancia del fondo de maniobra en el ámbito operativo empresarial.

Según criterios de Saucedo (2019) y Navarro (2018) el capital de trabajo es: "Inversión de una empresa en activos a corto plazo: efectivo, valores negociables, cuentas por cobrar e inventarios. El capital de trabajo según el proceso se divide en capital permanente o temporal. El capital permanente implica los requerimientos circulantes a largo plazo, a diferencia del temporal que se direcciona a las necesidades estacionarias. Gitman \& Zutter, (2012) y Ross, Westerfield y Jaffe (2012) mencionan que cuanto mayor sea el margen con el que los activos corrientes de una compañía sobrepasan a sus pasivos corrientes, mayor será la capacidad de esta para pagar sus cuentas a medida que se llegan a vencer. 
Si bien es cierto diversos autores, Van Horne \& Wachowicz (2010) y Jiménez, Rojas, \& Ospina (2013) concuerdan en que el capital de trabajo neto es una necesidad sumamente relevante para el desarrollo de las actividades operativas del negocio, por cuanto se hace imperioso la inducción de una eficiente administración de la misma. Según Hamid, et al. (2017) consideran que la administración del fondo de maniobra desempeña un factor vital para las operaciones cotidianas en la empresa, haciendo referencia a tres decisiones elementales (Estructura de capital, Presupuesto de capital y gestión de capital de trabajo).

Si el capital de trabajo neto es alto, implica una disminución del riesgo, pues se determina que la empresa dispone de los recursos necesarios para cubrir las obligaciones en el corto plazo, Córdova (2013). De igual forma es indispensable identificar las políticas de capital de trabajo que pueden establecerse en el ámbito empresarial. Según Espinoza, (2007) y Córdova (2007), las políticas del capital de trabajo se asocian estrechamente a las decisiones que toman los administradores financieros con respecto a los activo y pasivo circulantes. Estos niveles tienen un impacto directo en el riesgo y rentabilidad.

Existen diversos métodos para calcular el capital de trabajo: El método contable, en contexto general es el más utilizado y se establece mediante la diferencia entre los activos y pasivos circulantes. Duran (2011) manifiesta que se debe analizar el resultado que se va obtener, puesto que dependiendo si es positivo o negativo, se interpretará y se tomaran las decisiones pertinentes. Faxas del Toro \& Atucha (2011) consideran que se debe tener un equilibrio entre los activos y pasivos corrientes, puesto que la rentabilidad de la empresa puede depender de la fluctuación o comportamiento de dichas variables.

Para Aguilar (2017) otro método utilizado es el del ciclo de efectivo, básicamente es empleado para estimar el monto de efectivo necesario para la operación del negocio, especialmente es su etapa de inicio. Para su cálculo se hace referencia esencialmente al análisis del ciclo de caja, el mismo que se determina considerando el tiempo en días que la empresa demora en recuperar sus ingresos provenientes de las ventas, para cumplir con las obligaciones de proveedores. En este método al capital de trabajo también se lo conoce como efectivo mínimo de operaciones. Otro método identificado, hace referencia a los porcentajes de cambio de las ventas.

Desde una perspectiva de aplicación técnica porcentual se establecen dos escenarios para calcular la necesidad de fondos, cuando la organización desarrolla sus actividades al $100 \%$ de la capacidad instalada, tendrá que realizar inversiones de capital para poder incrementar la producción. En otro contexto si la entidad opera por debajo de su capacidad instalada, evidentemente direccionará esfuerzos a la inversión de activos corrientes para incrementar los ingresos. (Block, Hirt, \& Danielsen, 2013). En este ámbito Gonzales \& Quizhpe, (2012) establecen que una empresa para impulsar su crecimiento requiere de financiamiento para incrementar las ventas, por consiguiente, es imperioso la realización de inversiones 
tanto en cuentas por cobrar, inventarios etc. Es importante destacar que este método se caracteriza por las variaciones de las cuentas: efectivo en caja, clientes, existencias y proveedores (Porlles et al., 2013).

\subsection{ANÁLISIS DE LA RENTABILIDAD EN EL CONTEXTO EMPRESARIAL}

Soriano (2010) menciona que la rentabilidad es el resultado y/o beneficio que tienen las instituciones de lo invertido en relación a todos los componentes que fueron empleados en sus operaciones. Para Baena (2014) la rentabilidad es la obtención de resultados de una inversión en función de la rotación de los activos propiedad planta y equipo (conocidos como activos fijos), y el margen de utilidad neta.

Gitman et al. (2012) Consideran que la rentabilidad es: "la relación entre ingresos y costos generados por el uso de los activos de la empresa en actividades productivas. La rentabilidad de una empresa puede ser evaluada en referencia a las ventas, a los activos, al capital o al valor accionario" (p. 63). Abad (2011) considera que la rentabilidad se establece en un tiempo determinado, haciendo un análisis específico de la capacidad de generación de utilidades y por consiguiente la obtención de rendimientos sobre la inversión estipulada. Sin embargo, Van Horne \& Wachowicz (2010) menciona que, al buscar una rentabilidad más alta, se debe esperar mayores riesgos en la empresa pues se puede decir que el riesgo y el rendimiento van de la mano.

Según Zamora (2008) la rentabilidad implica una medición objetiva del rendimiento resultante de la operacionalización de los capitales utilizados en un periodo de tiempo específico. Para Córdova (2013) La rentabilidad se logra "aumentando los ingresos por medio de las ventas o disminuyendo los costos pagando menos por las materias primas, salarios o servicios que se presten” (p. 188).

Para Contreras (2015) y Cordova (2014) la rentabilidad económica muestra la eficacia en el uso de los activos, se mide dividiendo el beneficio operativo después de impuestos entre el activo neto promedio. La rentabilidad financiera o también llamado ratio de retorno determina la rentabilidad con respecto al patrimonio que se mide dividiendo la utilidad neta sobre patrimonio. Según Eslava (2010) Las dimensiones de rentabilidad se distinguen entre la rentabilidad económica o rentabilidad de la empresa como negocio y entre la rentabilidad financiera o rentabilidad que obtengan los accionistas o propietarios como retribución al capital que tiene invertido en la empresa.

Según García, Martinez, \& Fernandez (2018) consideran que la rentabilidad sobre activos mide la rentabilidad económica, este hace referencia a las utilidades antes de intereses e impuestos con los activos. Para Redondo \& Arias (2013), la rentabilidad sobre activos es: "La relación entre beneficios netos y activos totales. Representa la rentabilidad de la empresa respecto al activo invertido ya esté financiado con fondos propios o ajenos." 


\subsection{EL SECTOR MANUFACTURERO ECUATORIANO}

Las características que definen la estructura productiva de un país, sostenibilidad y el grado de especialización de los subsectores o actividades económicas que la integran son fundamentales para el desarrollo de la producción de su economía. La industria manufacturera cumple un factor preponderante en este ámbito, y a nivel de comparación este sector desarrolla actividades que representan un mayor crecimiento económico, progreso y cambio estructural de una nación, según informes de CEPAL, 2012.

Es importante recalcar que el sector manufacturero ecuatoriano según la Superintendencia de Compañías (2020) está subclasificada por subsectores, haciendo referencia a la Clasificación Industrial de Clasificación Uniforme (CIIU), totalizando a 24 categorias de empresas. Estas compañías desarrolan actividades operativas diferentes, tienen tamaños disimiles, no obstante constituyen un pilar fundamental para el desarrollo económico del pais.

Durante el periodo 2013-2017 la industria manufacturera generó 5,941 millones de USD en utilidades (1,098 millones de USD en promedio anual). Los ingresos por ventas para fueron aproximadamente de 120,871 millones de USD (24,174 millones de USD en promedio anual). Mediante un análisis de incidecia regional en el periodo 2017, Pichincha reporta 9,443 millones de USD y una participación del $41.2 \%$ en el total de ingresos por ventas obtenidos por el sector manufacturero a nivel nacional, Guayas es la provincia que sigue con 8,724 millones de USD y una participación del 38\%. Manabí reportó 1,843 millones de USD y una participación del 8\%, Y Azuay con un total de 1,175 millones de USD en ingresos por ventas y una participación del 5.1\%, instituyendose como las provincias de mayor representatividad en el ambito de la industria manufacturera. (Superintendencia de Compañías, 2018).

\section{MATERIALES Y MÉTODOS}

La presente investigación se direcciona mediante la inducción de un enfoque cuantitativo, puesto que se requiere de la indagación específica de indicadores tanto en el ámbito de la gestión de capital de trabajo como en el estudio de las tendencias de rentabilidad de las empresas del sector manufacturero. Estos indicadores corresponden a las medianas por subsector de todas las compañías activas que reportaron su información financiera en los últimos cinco años, hasta el cierre del periodo 2018.

El diseño de investigación se conjetura como no experimental longitudinal, debido a que se pretende generar un análisis fehaciente recopilando información en diversos periodos respecto a las variables de estudio (Capital de trabajo - Rentabilidad). Se procederá a la aplicación del tipo de investigación descriptivo, explicativa y correlacional. El estudio se realizó al sector total, el área de 


\begin{tabular}{|c|c|c|}
\hline $\mathrm{N}^{\mathrm{o}}$ & DESCRIPCIÓN & ROA \\
\hline 1 & C10 - Elaboración de productos alimenticios. & 0,0065 \\
\hline 2 & C12 - Elaboración de productos de tabaco. & 0,0975 \\
\hline 3 & C13 - Fabricación de productos textiles. & 0,0226 \\
\hline 4 & C14 - Fabricación de prendas de vestir. & 0,011 \\
\hline 5 & C15 - Fabricación de cueros y productos conexos. & 0,0285 \\
\hline 6 & $\begin{array}{l}\text { C16 - Producción de madera y fabricación de productos de madera y } \\
\text { corcho, excepto muebles; fabricación de artículos de paja y de } \\
\text { materiales trenzables. }\end{array}$ & 0,0111 \\
\hline 7 & C17 - Fabricación de papel y de productos de papel. & 0,0267 \\
\hline 8 & C18 - Impresión y reproducción de grabaciones. & 0,0207 \\
\hline 9 & C20 - Fabricación de substancias y productos químicos. & 0,0225 \\
\hline 10 & $\begin{array}{l}\text { C21 - Fabricación de productos farmacéuticos, sustancias químicas } \\
\text { medicinales y productos botánicos de uso farmacéutico. }\end{array}$ & 0,0321 \\
\hline 11 & C22 - Fabricación de productos de caucho y plástico. & 0,026 \\
\hline 12 & C23 - Fabricación de otros productos minerales no metálicos. & 0,01 \\
\hline 13 & C24 - Fabricación de metales comunes. & 0,0197 \\
\hline 14 & $\begin{array}{l}\text { C25 - Fabricación de productos elaborados de metal, excepto } \\
\text { maquinaria y equipo. }\end{array}$ & 0,0208 \\
\hline 15 & C26 - Fabricación de productos de informática, electrónica y óptica. & 0,0079 \\
\hline 16 & C27 - Fabricación de equipo eléctrico. & 0,0219 \\
\hline 17 & C28 - Fabricación de maquinaria y equipo n.c.p. & 0,0257 \\
\hline 18 & $\begin{array}{l}\text { C29 - Fabricación de vehículos automotores, remolques y } \\
\text { semirremolques. }\end{array}$ & 0,0455 \\
\hline 19 & C30 - Fabricación de otros tipos de equipos de transporte. & 0,0199 \\
\hline 20 & C33 - Reparación e instalación de maquinaria y equipo. & 0,0331 \\
\hline
\end{tabular}

Fuente: Superintendencia de Compañías (2020)

Se procedió a la aplicación de análisis estadístico descriptivo para establecer para cado estado financiero, las medidas de tendencia centrales y de dispersión y posición, respecto al tratamiento de los indicadores suscritos en el estudio pertinente, la estadística inferencial y coeficiente de correlación y determinación. Se aplicó un análisis multivariante con base a un modelo de regresión lineal múltiple para establecer específicamente el nivel de relación de los diversos indicadores capital de trabajo (Variable Independiente) en contexto con la rentabilidad (variable dependiente), permitiendo generar un resultado concluyente en torno al proceso investigativo. El modelo pretende establecer R cuadrado (Coeficiente de determinación) por periodo estudiado, posteriormente se aplicará la prueba de bondad de ajuste como el 
análisis de la varianza(Anova) para comprobar el nivel de relación de del modelo de regresión (F) y estipular la significancia (Sig) de acuerdo a los residuos y de igual manera la prueba t de student al 5\% de significancia.

\section{RESULTADOS}

\subsection{DIAGNÓSTICO SOBRE LA GESTIÓN DE CAPITAL DE TRABAJO DE LAS EMPRESAS DEL} SECTOR MANUFACTURERO ECUATORIANO AL CIERRE DEL PERIODO 2018

Para el desarrollo del diagnóstico de la gestión de capital de trabajo se establece las medias por año de los indicadores: Rotación de cartera, rotación de activo fijo, rotación de ventas, periodo medio de cobranza y periodo medio de pago. Como se puede observar en la tabla 2, en los años 2014, 2015 y 2017 en promedio se genera una mayor eficiencia en la gestión de capital de trabajo, puesto que los indicadores son más fehacientes en esos periodos.

Tabla 2. Medias - Indicadores GCT

\begin{tabular}{|c|c|c|c|c|c|}
\hline Año & $\begin{array}{c}\text { Rotación De } \\
\text { Cartera }\end{array}$ & $\begin{array}{c}\text { Rotación De } \\
\text { Activo Fijo }\end{array}$ & $\begin{array}{l}\text { Rotación De } \\
\text { Ventas }\end{array}$ & $\begin{array}{l}\text { Periodo Medio De } \\
\text { Cobranzas (Días) }\end{array}$ & $\begin{array}{l}\text { Periodo Medio De } \\
\text { Pago (Días) }\end{array}$ \\
\hline 2014 & 3,77708 & 2,91866 & 1,09928 & 45,22389 & 83,138845 \\
\hline 2015 & 3,444965 & 2,52317 & 1,053985 & 47,290685 & 77,758625 \\
\hline 2016 & 2,213215 & 1,031465 & 0,769785 & 48,379205 & 0 \\
\hline 2017 & 3,5673 & 3,97266 & 0,925175 & 85,56235 & 329,584315 \\
\hline 2018 & 2,767425 & 1,8631 & 0,926695 & 65,358285 & 121,670525 \\
\hline
\end{tabular}

Fuente: Tabla de indicadores - Superintendencia de Compañías - Procesamiento SPSS

\subsection{NIVEL DE RENTABILIDAD DE LAS DE LAS EMPRESAS DEL SECTOR MANUFACTURERO.}

Se procede al análisis de las Medias de la Rentabilidad del activo de los subsectores del sector manufacturero por año, con el propósito de identificar fluctuaciones más altas en los años estudiados. En el año 2017 se observa un nivel mayor de rentabilidad del activo (0,0498). Al contrastar esta información con los factores promédiales anuales de gestión de capital de trabajo, se puede suponer que en dicho periodo la gestión de capital de trabajo fue eficiente, además el periodo medio de pago corresponde a la más alta fluctuación. Los subsectores con mayor rentabilidad según muestra la tabla 3 corresponden a la fabricación de vehículos automotores remolques y semirremolques (C29). Y reparación e instalación de maquinaria y equipo. (C33) con una media similar de 0,0406 y 0,0473 respectivamente.

Tabla 3. ROA promedio por sector - periodo 2014 - 2018

\begin{tabular}{lrrrrrrrrrrr}
\hline & \multicolumn{1}{c}{ C10 } & \multicolumn{1}{c}{ C12 } & C13 & C14 & C15 & C16 & C17 & C18 & C20 & C21 \\
\hline N Válido & 5 & 5 & 5 & 5 & 5 & 5 & 5 & 5 & 5 & 5 \\
$\quad$ Perdidos & 0 & 0 & 0 & 0 & 0 & 0 & 0 & 0 & 0 & 0 \\
Media &, 0197 &, 0621 &, 0242 &, 0187 &, 0190 &, 0213 &, 0301 &, 0327 &, 0313 &, 0312 \\
Mediana &, 0228 &, 0756 &, 0226 &, 0169 &, 0262 &, 0228 &, 0267 &, 0288 &, 0275 &, 0321
\end{tabular}




\begin{tabular}{|c|c|c|c|c|c|c|c|c|c|c|}
\hline $\begin{array}{l}\text { Desviación } \\
\text { estándar }\end{array}$ & ,01674 & ,04045 & ,01600 & ,01329 & ,01202 & ,01463 & ,01691 & ,01418 & ,01937 & ,02237 \\
\hline Mínimo &, 00 &, 00 &, 00 & ,00 & ,00 &, 00 & ,01 & ,02 &, 01 & ,00 \\
\hline Máximo &, 04 &, 10 &, 04 &, 04 & ,03 &, 04 &, 06 &, 05 &, 06 &, 06 \\
\hline & $\mathrm{C} 22$ & $\mathrm{C} 23$ & $\mathrm{C} 24$ & $\mathrm{C} 25$ & $\mathrm{C} 26$ & $\mathrm{C} 27$ & $\mathrm{C} 28$ & $\mathrm{C} 29$ & C30 & C33 \\
\hline Válido & 5 & 5 & 5 & 5 & 5 & 5 & 5 & 5 & 5 & 5 \\
\hline Perdidos & 0 & 0 & 0 & 0 & 0 & 0 & 0 & 0 & 0 & 0 \\
\hline Media & 0371 & ,0204 & 0253 & 0390 & ,0263 & ,0317 & ,0303 & ,0406 & ,0184 &, 0473 \\
\hline Mediana & ,0431 & ,0189 & ,0197 &, 0356 & ,0187 & ,0374 &, 0257 & ,0396 & ,0159 &, 0575 \\
\hline $\begin{array}{l}\text { Desviación } \\
\text { estándar }\end{array}$ & ,01544 & ,01619 & ,01258 & ,01649 & ,02473 & ,01286 & ,02380 & ,01787 & ,00923 & ,01903 \\
\hline Mínimo & ,02 &, 00 &, 01 &, 02 & ,00 &, 01 &, 00 & ,02 & ,01 & ,02 \\
\hline Máximo &, 05 &, 04 & ,04 & ,06 &, 05 &, 04 &, 07 & ,07 & ,03 & ,06 \\
\hline
\end{tabular}

Fuente: Tabla de indicadores - Superintendencia de Compañías - Procesamiento SPSS

\subsection{RELACIÓN ENTRE LA GESTIÓN DE CAPITAL DE TRABAJO Y LA RENTABILIDAD}

\subsubsection{Modelo de regresión lineal múltiple (Análisis Longitudinal)}

Para el periodo 2014 el modelo toma como referencia los siguientes predictores: (Constante): Periodo medio de cobranza, rotación de cartera, rotación de activo fijo, rotación de ventas, en relación a la variable dependiente: Rentabilidad neta del activo. R Cuadrado indica el porcentaje de incidencia de las variables independientes en torno a la variable dependiente. Se procede a la aplicación de ANOVA para determinar la significancia del modelo en función a los predictores establecidos. En la tabla Resumen 17, se muestra la Matriz general de resultados por año (R Cuadrado - Anova).

Tabla 4. Coeficientes variables independientes - periodo 2014

\begin{tabular}{|c|c|c|c|c|c|c|c|c|}
\hline \multirow{2}{*}{\multicolumn{2}{|c|}{ Modelo }} & \multicolumn{2}{|c|}{$\begin{array}{l}\text { Coeficientes no } \\
\text { estandarizados }\end{array}$} & $\begin{array}{l}\text { Coeficientes } \\
\text { estandarizados }\end{array}$ & \multirow[b]{2}{*}{$\mathrm{T}$} & \multirow[b]{2}{*}{ Sig. } & \multicolumn{2}{|c|}{$\begin{array}{l}95.0 \% \text { intervalo de } \\
\text { confianza para B }\end{array}$} \\
\hline & & $\mathrm{B}$ & $\begin{array}{l}\text { Error } \\
\text { estándar }\end{array}$ & Beta & & & $\begin{array}{l}\text { Limite } \\
\text { inferior }\end{array}$ & $\begin{array}{c}\text { Limite } \\
\text { superior }\end{array}$ \\
\hline 1 & (Constante) &,- 038 & ,025 & & $-1,487$ &, 159 &,- 093 & ,017 \\
\hline & $\mathrm{RC}$ & ,000 & ,004 & ,006 & ,029 & ,977 &,- 009 & ,009 \\
\hline & RAF &,- 005 & ,005 &,- 232 & $-1,030$ & ,320 &,- 016 & ,006 \\
\hline & RV & ,039 & ,028 & ,372 & 1,355 & , 197 &,- 022 & , 100 \\
\hline & PMC &, 001 &, 000 & ,854 & 4,314 &, 001 &, 000 & ,001 \\
\hline
\end{tabular}

Fuente: Resultados obtenidos - Procesamiento SPSS

Con base al análisis de coeficientes se procede a introducir en el modelo únicamente a la variable independiente Periodo Medio de Cobranza (PMC) puesto que es el indicador más significativo.

Tabla 5. Anova - Periodo medio de Cobranza - periodo 2014

\begin{tabular}{llrrrrr}
\hline Modelo & & Suma de cuadrados & Gl & Media cuadrática & F & Sig. \\
\hline 1 & Regresión &, 004 & 1 &, 004 & 18,189 &, $001^{\text {b }}$ \\
& Residuo &, 003 & 17 &, 000 & \\
\hline \multicolumn{2}{l}{ Total } &, 007 & 18 & & \\
\hline
\end{tabular}

Fuente: Resultados obtenidos - Procesamiento SPSS 
Tabla 6. Coeficientes - Periodo medio de Cobranza - periodo 2014

\begin{tabular}{|c|c|c|c|c|c|c|c|c|}
\hline \multirow{2}{*}{\multicolumn{2}{|c|}{ Modelo }} & \multicolumn{2}{|c|}{$\begin{array}{l}\text { Coeficientes no } \\
\text { estandarizados } \\
\text { Error }\end{array}$} & \multicolumn{2}{|l|}{$\begin{array}{l}\text { Coeficientes } \\
\text { estandarizados }\end{array}$} & \multirow[b]{2}{*}{ Sig. } & \multicolumn{2}{|c|}{$\begin{array}{l}95.0 \% \text { intervalo de } \\
\text { confianza para B }\end{array}$} \\
\hline & & B & $\begin{array}{l}\text { Error } \\
\text { estándar }\end{array}$ & Beta & $\mathrm{t}$ & & $\begin{array}{l}\text { Limite } \\
\text { inferior }\end{array}$ & $\begin{array}{l}\text { Limite } \\
\text { superior }\end{array}$ \\
\hline 1 & (Constante) &,- 004 &, 009 & &,- 426 &, 675 &,- 022 & ,014 \\
\hline & $\begin{array}{l}\text { Período medio de } \\
\text { cobranza }\end{array}$ & ,001 &, 000 &, 719 & 4,265 & ,001 &, 000 & ,001 \\
\hline
\end{tabular}

Fuente: Resultados obtenidos - Procesamiento SPSS

En el periodo 2014 se establece que el modelo global es significadito, es decir las variables independientes explican a la dependiente, no obstante, en el análisis por variable se visualiza que el periodo medio de cobranza representa un mayor nivel de significación, según se evidencia resultados en tabla 5 y 6 , por cuanto mediante la aplicación del modelo se determina un R cuadrado de $0,517, \mathrm{~F} 18,189$ y Sig. 0,01. Es decir, la variable periodo medio de cobranza explica a la rentabilidad del activo en un $51,7 \%$ considerando que la relación según Anova es sustancial.

Continuando el análisis en el período 2015 según los coeficientes de correlación se estabelece que existe uma asociación baja - moderada entre las variables rentabilidad del activo y las variables PMC PMP. El modelo global para el periodo 2015 aplicado para los predictores periodos medio de pago, rotación de cartera, rotación de activo fijo, rotación de ventas, periodo medio de cobranza es significativo en relación a la rentabilidad del activo.

Tabla 7. Coeficientes variables independientes - periodo 2015

\begin{tabular}{|c|c|c|c|c|c|c|c|c|}
\hline & & \multicolumn{2}{|c|}{$\begin{array}{l}\text { Coeficientes no } \\
\text { estandarizados }\end{array}$} & \multicolumn{2}{|l|}{$\begin{array}{l}\text { Coeficientes } \\
\text { estandarizados }\end{array}$} & \multirow[b]{2}{*}{ Sig. } & \multicolumn{2}{|c|}{$\begin{array}{l}95.0 \% \text { intervalo de } \\
\text { confianza para B }\end{array}$} \\
\hline \multicolumn{2}{|c|}{ Modelo } & B & $\begin{array}{l}\text { Error } \\
\text { estándar }\end{array}$ & Beta & $\mathrm{T}$ & & $\begin{array}{l}\text { Limite } \\
\text { inferior }\end{array}$ & $\begin{array}{l}\text { Limite } \\
\text { superior }\end{array}$ \\
\hline 1 & (Constante) &,- 054 &, 021 & & $-2,624$ &, 020 &,- 098 &,- 010 \\
\hline & $\mathrm{RC}$ &,- 001 & ,003 &,- 076 &,- 443 & ,665 &,- 008 &, 005 \\
\hline & RA &,- 001 &, 003 &,- 039 &,- 227 & ,824 &,- 008 & ,006 \\
\hline & $\mathrm{RV}$ & ,068 &, 024 & ,671 & 2,781 & ,015 & ,016 & , 120 \\
\hline & PMC & $-1,436 \mathrm{E}-5$ &, 000 &,- 014 &,- 052 & ,959 &,- 001 & ,001 \\
\hline & PMP &, 00024 & ,000 & 1,103 & 3,437 &, 004 &, 000 &, 0004 \\
\hline
\end{tabular}

Fuente: Resultados obtenidos - Procesamiento SPSS

Como se observa en el análisis de coeficientes del modelo global la rotación de ventas y Periodo medio de pago tienen significancia, por cuanto se procede a desarrollar el modelo analizando específicamente estas dos variables.

Tabla 8. Resumen del modelo - Periodo medio de Pago - Rotación de ventas - periodo 2015

\begin{tabular}{lrrrr}
\hline & & & \multicolumn{2}{c}{$\begin{array}{c}\text { Error estándar de la } \\
\text { estimación }\end{array}$} \\
\hline 1 & $\mathrm{R}$ & R cuadrado & R cuadrado ajustado &, 0137680 \\
2 &, $720^{\text {a }}$ &, 519 &, 492 &, 0102202 \\
\hline
\end{tabular}


a. Predictores: (Constante), PERIODO MEDIO DE PAGO

b. Predictores: (Constante), ROTACION DE VENTAS

Fuente: Resultados obtenidos - Procesamiento SPSS

Tabla 9. Anova - Periodo medio de Pago - Rotación de ventas - periodo 2015

\begin{tabular}{llrrrrr}
\hline Modelo & & Suma de cuadrados & Gl & Media cuadrática & F & Sig. \\
\hline 1 & Regresión &, 004 & 1 &, 004 & 19,418 &, $000^{\mathrm{b}}$ \\
& Residuo &, 003 & 18 &, 000 & \\
& Total &, 007 & 19 & &, $000^{\mathrm{c}}$ \\
2 & Regresión &, 005 & 2 &, 003 & 25,453 & \\
& Residuo &, 002 & 17 &, 000 & \\
& Total &, 007 & 19 & & \\
\hline
\end{tabular}

b. Predictores: (Constante), Período medio de pago

c. Predictores: (Constante), Rotación de ventas

Fuente: Resultados obtenidos - Procesamiento SPSS

Tabla 10. Coeficientes - Periodo medio de pago - Rotación de ventas - periodo 2015

\begin{tabular}{|c|c|c|c|c|c|c|c|c|}
\hline & & \multicolumn{2}{|c|}{$\begin{array}{r}\text { Coeficientes no } \\
\text { estandarizados } \\
\text { Error }\end{array}$} & \multicolumn{2}{|l|}{$\begin{array}{l}\text { Coeficientes } \\
\text { estandarizados }\end{array}$} & & \multicolumn{2}{|c|}{$\begin{array}{c}95.0 \% \text { intervalo de } \\
\text { confianza para B }\end{array}$} \\
\hline \multicolumn{2}{|c|}{ Modelo } & B & $\begin{array}{l}\text { Error } \\
\text { estándar }\end{array}$ & Beta & $\mathrm{T}$ & Sig. & $\begin{array}{l}\text { Limite } \\
\text { inferior }\end{array}$ & $\begin{array}{l}\text { Limite } \\
\text { superior }\end{array}$ \\
\hline \multirow[t]{2}{*}{1} & (Constante) &, 017 &, 004 & & 4,118 &, 001 &, 008 & , 026 \\
\hline & $\begin{array}{l}\text { Período medio de } \\
\text { pago }\end{array}$ & ,00016 & ,000 & ,720 & 4,407 & ,000 & ,000 & ,00023 \\
\hline \multirow[t]{2}{*}{2} & (Constante) &,- 051 & 017 & & $-2,921$ & 010 &,- 088 &,- 014 \\
\hline & Rotación de ventas & 059 & .015 &, 585 & 3,958 & .001 & .028 & ,091 \\
\hline
\end{tabular}

En el periodo 2015 se establece que el modelo global es significadito, es decir las variables independientes explican a la dependiente, sin embargo, en el estudio de coeficientes por variable se visualiza que el periodo medio de pago y la rotación de ventas representa un mayor nivel de significación, según resultados de tabla 9 y 10 , por cuanto mediante la aplicación del modelo se determina que el R cuadrado más alto corresponde a rotación de ventas con el de 0,750, F de 25,453 y Sig. 0,000. Es decir, la variable Rotación de ventas explica a la rentabilidad del activo en un 75,5\% considerando que la relación según Anova es sustancial.

En el período 2016 para proceder al desarrollo del modelo es pertinente recalcar que la variable Periodo medio de pago fue extraída del estudio debido a la insuficiencia de datos. De igual manera bajo esa misma premisa se excluyó el subsector C12 - C11 - C 23, puesto que la variable dependiente (Rentabilidad) para estos tres casos es cero según el informe emitido por la Superintendencia de Compañías. En el periodo 2016 se observa mediante la correlación de Pearson un nivel de asociación considerable de la variable dependiente (Rentabilidad del activo) y la variable independiente (Rotación de ventas). Con base a lo expuesto se procede a la aplicación del modelo. El modelo global es significativo, considerando un indicador de Fisher de 4,822 y un nivel de significancia de 0,014, menor a 0,05e puede 
deducir según $\mathrm{R}$ cuadrado que las variables independientes (Indicadores de rentabilidad) explican a la variable dependiente (Rentabilidad del activo) en un 61,9\%.

Tabla 11. Coeficientes variables independientes - periodo 2016

\begin{tabular}{|c|c|c|c|c|c|c|c|c|}
\hline \multirow{2}{*}{\multicolumn{2}{|c|}{ Modelo }} & \multicolumn{2}{|c|}{$\begin{array}{l}\text { Coeficientes no } \\
\text { estandarizados }\end{array}$} & \multicolumn{3}{|l|}{$\begin{array}{l}\text { Coeficientes } \\
\text { estandarizados }\end{array}$} & \multicolumn{2}{|c|}{$\begin{array}{l}95.0 \% \text { intervalo de } \\
\text { confianza para B }\end{array}$} \\
\hline & & B & $\begin{array}{l}\text { Error } \\
\text { estándar }\end{array}$ & Beta & $\mathrm{T}$ & Sig. & $\begin{array}{l}\text { Limite } \\
\text { inferior }\end{array}$ & $\begin{array}{l}\text { Limite } \\
\text { superior }\end{array}$ \\
\hline 1 & (Constante) &,- 018 & ,009 & & $-2,161$ &, 052 &,- 037 & ,000 \\
\hline & $\mathrm{RC}$ & ,001 & ,003 & ,061 & ,241 & ,814 &,- 005 & ,007 \\
\hline & RAF & ,000 & ,003 & ,049 & , 174 & ,865 &,- 005 & ,006 \\
\hline & $\mathrm{RV}$ &, 031 & 010 & ,705 & 3,005 & ,011 & ,009 &, 054 \\
\hline & PMC & $1,950 \mathrm{E}-5$ & ,000 & ,039 &, 190 & ,852 & ,000 & ,000 \\
\hline
\end{tabular}

Como se puede observar en tabla 11, efectuando un análisis de coeficientes la variable significativa corresponde a Rotación de ventas tiene mayor significancia, por cuanto se procede a efectuar el modelo de regresión para dicha variable, con el propósito de medir su nivel de incidencia en la rentabilidad. En el periodo 2016 la variable rotación de ventas genera una mayor incidencia en la variable rentabilidad del activo, el factor de Fisher es de 23,428 y el nivel de significancia es mucho menor a 0,05.

En el período 2017 se puede observar una asociación directa de tipo moderada entre la variable rotación de ventas y rentabilidad del activo. Predictores: Periodo medio de pago, rotación de activo fijo, rotación de ventas, rotación de cartera, periodo medio de cobranza. Según los resultados evidenciados, el modelo global es significativo, no obstante, se puede considerar que para este periodo la relación de las variables independientes y la variable dependiente no es sustancial, esto se corrobora con la identificación de la prueba de Fisher $(3,198)$.

Tabla 12. Coeficientes variables independientes - periodo 2017

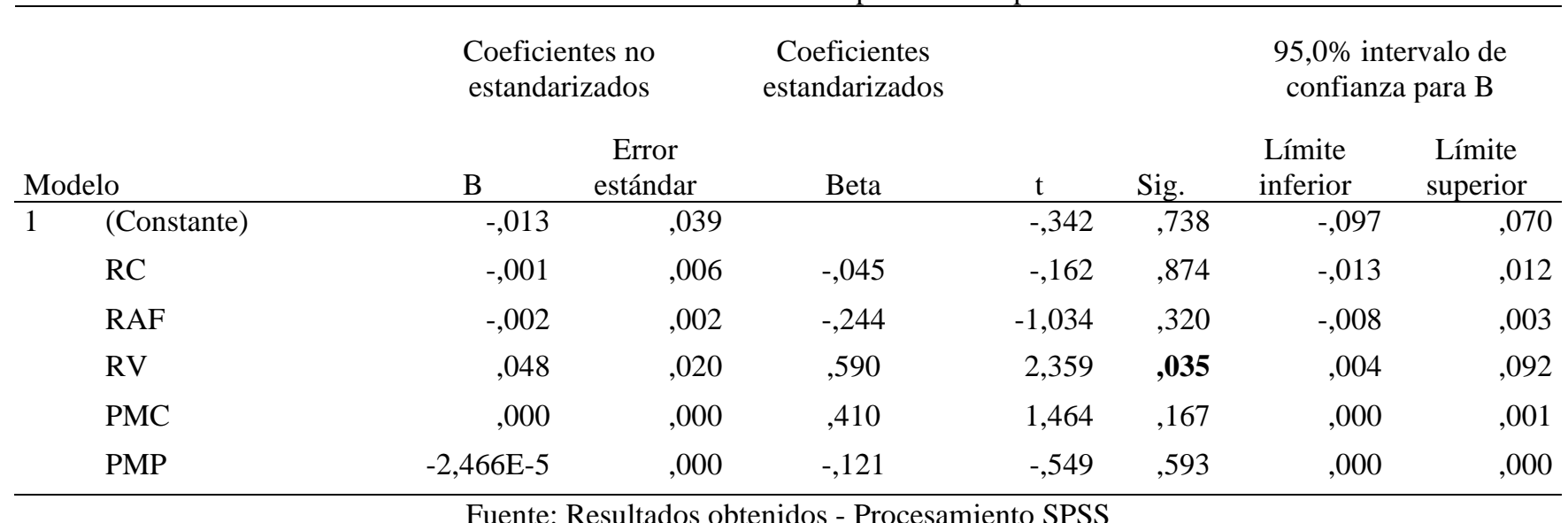

Fuente: Resultados obtenidos - Procesamiento SPSS 
Como se puede observar en tabla 12, la variable independiente significativa es rotación de ventas, por cuanto se procede a establecer el modelo específicamente para esta variable, con el propósito de conocer por medio de R cuadrado su nivel de incidencia en la rentabilidad del activo. Según la prueba de Fisher en 11,888, la variable independiente Rotación de ventas tiene una relación considerable con la rentabilidad del activo. La rotación de ventas para el periodo 2017 explica en un 41,2\% a la variable dependiente rentabilidad del activo.

Tabla 13. Resumen del modelo - Rotación de ventas - periodo 2017

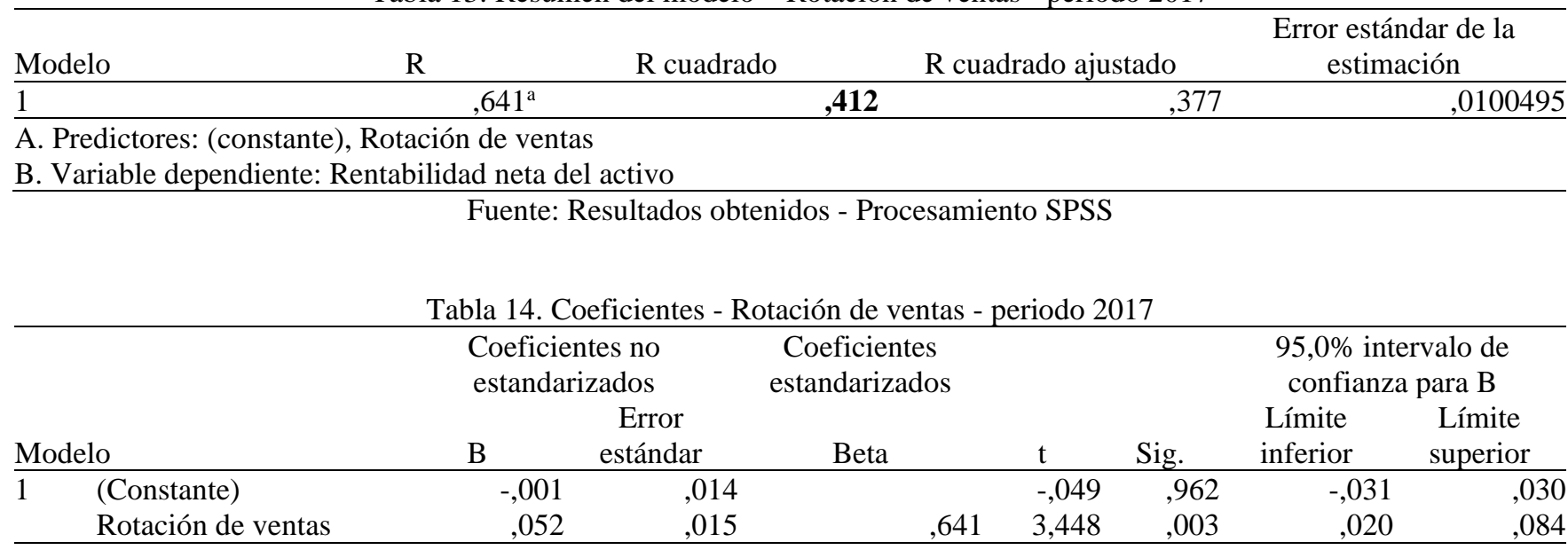

Fuente: Resultados obtenidos - Procesamiento SPSS

Para el análisis del periodo 2018 se procedió a eliminar los casos C30 (fabricación de otros tipos de equipos de transporte) y C33 (reparación e instalación de maquinaria y equipo) por falta de datos. El coeficiente de Pearson establece un nivel de asociación bajo - moderado con de los indicadores Rotación de activo fijo y Periodo medio de cobranza con la Rentabilidad del activo. Según los resultados establecidos el modelo global para el periodo 2018 no es significativo, por cuanto se procederá al análisis de la variable de mayor proximidad a la significancia, de acuerdo a al análisis de coeficientes:

Tabla 15. Coeficientes variables independientes - periodo 2018

\begin{tabular}{|c|c|c|c|c|c|c|c|c|}
\hline & & \multicolumn{2}{|c|}{$\begin{array}{l}\text { Coeficientes no } \\
\text { estandarizados }\end{array}$} & \multicolumn{2}{|l|}{$\begin{array}{l}\text { Coeficientes } \\
\text { estandarizados }\end{array}$} & & \multicolumn{2}{|c|}{$\begin{array}{c}95.0 \% \text { intervalo de } \\
\text { confianza para B }\end{array}$} \\
\hline \multicolumn{2}{|c|}{ Modelo } & B & $\begin{array}{l}\text { Error } \\
\text { estándar }\end{array}$ & Beta & $\mathrm{T}$ & Sig. & $\begin{array}{l}\text { Limite } \\
\text { inferior }\end{array}$ & $\begin{array}{l}\text { Limite } \\
\text { superior }\end{array}$ \\
\hline 1 & (Constante) &,- 020 &, 038 & &,- 532 &, 605 &,- 104 &, 063 \\
\hline & $\mathrm{RC}$ &, 005 & ,013 & , 129 & ,390 & ,704 &,- 024 & ,035 \\
\hline & RAF & ,019 & 010 & ,673 & 1,972 & ,072 &,- 002 & 041 \\
\hline & RV &,- 058 &, 055 &,- 396 & $-1,050$ & ,314 &,- 178 & ,062 \\
\hline & PMC &, 001 & ,000 &, 423 & 1,399 & 187 & ,000 &, 002 \\
\hline & PMP & $3,853 \mathrm{E}-5$ &, 000 &, 118 &, 503 & 624 &, 000 &, 000 \\
\hline
\end{tabular}

Fuente: Resultados obtenidos - Procesamiento SPSS 
Como se puede observar la variable que mayor aproximación tiene al nivel de significancia es la Rotación de Activo Fijo, por cuanto se procede a establecer específicamente para dicha variable, con el propósito de identificar si el coeficiente de determinación (R Cuadrado) es fehaciente. El coeficiente $\mathrm{R}$ Cuadrado es bajo en relación a lo esperado, no obstante, se podría establecer que la variable independiente (Rotación de Activo Fijo) explica a la variable dependiente (Rentabilidad del Activo) en un 38,7\%.

Tabla 16. Coeficientes - Rotación de activo fijo - periodo 2018

\begin{tabular}{|c|c|c|c|c|c|c|c|c|}
\hline \multirow{2}{*}{\multicolumn{2}{|c|}{ Modelo }} & \multirow{2}{*}{\multicolumn{2}{|c|}{$\begin{array}{r}\text { Coeficientes no } \\
\text { estandarizados } \\
\text { Error }\end{array}$}} & \multirow{2}{*}{\multicolumn{2}{|c|}{$\begin{array}{l}\text { Coeficientes } \\
\text { estandarizados }\end{array}$}} & \multirow[b]{2}{*}{ Sig. } & \multicolumn{2}{|c|}{$\begin{array}{l}95.0 \% \text { intervalo de } \\
\text { confianza para B }\end{array}$} \\
\hline & & & & & & & $\begin{array}{l}\text { Limite } \\
\text { inferior }\end{array}$ & $\begin{array}{c}\text { Limite } \\
\text { superior }\end{array}$ \\
\hline 1 & (Constante) &,- 010 & ,012 & &,- 848 &, 409 &,- 035 &, 015 \\
\hline & RAF & ,018 & ,006 & ,622 & 3,178 &, 006 & ,006 &, 030 \\
\hline
\end{tabular}

Fuente: Resultados obtenidos - Procesamiento SPSS

\subsection{MATRIZ GENERAL DE RESULTADOS POR AÑO (R CUADRADO - ANOVA) MODELO GLOBAL}

Tabla 17. Matriz general de resultados por año (R Cuadrado - Anova)

\begin{tabular}{|c|c|c|c|c|}
\hline \multirow{2}{*}{ AÑ } & \multirow{2}{*}{$\mathrm{R}$ cuadrado } & \multicolumn{2}{|c|}{ Anova } & \multirow{2}{*}{ Interpretación } \\
\hline & & $\mathrm{F}$ & Sig & \\
\hline 2014 & 0,592 & 5,078 & 0,01 & El modelo global es significativo \\
\hline 2015 & 0,754 & 8,593 & 0,001 & El modelo global es significativo \\
\hline 2016 & 0,619 & 4,882 & 0,014 & El modelo global es significativo \\
\hline 2017 & 0,552 & 3,198 & 0,042 & El modelo global es significativo \\
\hline 2018 & 0,528 & 2,683 & 0,075 & El modelo global no es significativo \\
\hline
\end{tabular}

Fuente: Resultados obtenidos - Procesamiento SPSS

\subsection{MATRIZ ESPECÍFICA DE RESULTADOS ANUALES POR VARIABLES DE ESTUDIO}

Tabla 18. Matriz de resultados por variable de Rotación de Activo F- Rotación de Ventas (R Cuadrado - Anova)

\begin{tabular}{|c|c|c|c|c|c|c|}
\hline \multirow{3}{*}{ AÑO } & \multicolumn{3}{|c|}{ Rotación de Activo F } & \multicolumn{3}{|c|}{ Rotación de Ventas } \\
\hline & \multirow{2}{*}{$\mathrm{R}^{2}$} & \multicolumn{2}{|c|}{ Anova } & \multirow{2}{*}{$\mathrm{R}^{2}$} & \multicolumn{2}{|c|}{ Anova } \\
\hline & & $\mathrm{F}$ & Sig. & & $\mathrm{F}$ & Sig. \\
\hline 2014 & \multicolumn{3}{|c|}{ No es significativa } & \multicolumn{3}{|c|}{ No es significativa } \\
\hline 2015 & \multicolumn{3}{|c|}{ No es significativa } & 0,75 & 25,453 & $\mathbf{0 , 0 0 0}$ \\
\hline 2016 & \multicolumn{3}{|c|}{ No es significativa } & $\mathbf{0 , 6 1}$ & 23,428 & $\mathbf{0 , 0 0 0}$ \\
\hline 2017 & \multicolumn{3}{|c|}{ No es significativa } & 0,412 & 11,888 & $\mathbf{0 , 0 0 3}$ \\
\hline 2018 & 0,387 & 10,101 & 0,006 & & es significa & \\
\hline
\end{tabular}

Según los resultados identificados en el análisis longitudinal se establece que los modelos son significativos para los periodos 2014, 2015, 2016, 2017, lo que significa que existe relación entre las variables independientes y la variable dependiente para esos años. Sin embargo, en el periodo 2018 el 
modelo no es significativo. La variable independiente que mayor incidencia genera en la rentabilidad del activo es la rotación de ventas, puesto que su significancia se replica en tres años consecutivos (2015, 2016, 2017).

\section{DISCUSIÓN Y CONCLUSIONES}

En el año 2017 en el sector manufacturero se identifica un mayor nivel de rentabilidad del activo $(0,0498)$ en relación a los otros periodos analizados, al referenciar y contrastar este resultado con los factores promediales de los indicadores de gestión de capital de trabajo, se visualiza una rotación de activo fijo muy superior $(3,97)$ por cuanto se supone existió un mejor aprovechamiento del activo fijo para obtener ingresos. El análisis de correlación por periodos con respecto a los indicadores de gestión de capital de trabajo y la rentabilidad del activo no identifican niveles de asociación importantes o tendencias constantes, sin embargo, se evidencia en los periodos 2016 -2017 una relación moderada directa entre la rotación de ventas y la rentabilidad del activo. Este resultado es aceptable debido a que la rotación de ventas determina la eficiencia directiva, por consiguiente, se direcciona al incremento de la rentabilidad.

La aplicación de regresión múltiple en el estudio longitudinal estableció que los modelos globales son significativos desde 2014 al 2017, esto implica que el nivel de gestión de los indicadores de capital de trabajo incide en la rentabilidad del activo. Estos hallazgos se alinean a los postulados expuestos por Garcìa, Martinez \& Fernandez (2018), Cuenca (2018) y Duran (2011), en donde se estipula la importancia de la gestión de capital de trabajo en la rentabilidad a nivel empresarial. En el periodo 2018 no se obtuvo significancia general, por cuanto no se encontró evidencia suficiente para establecer que en ese año la gestión de capital de trabajo afectó la rentabilidad del activo de manera global.

Mediante la aplicación específica del modelo para las variables predictores de mayor significancia, se identificó que la gestión de la rotación de ventas incide en la rentabilidad del activo en tres años continuos $(2015,2016,2017)$, Por cuanto se puede inferir que para generar un nivel aceptable de rentabilidad del activo los administradores de las empresas del sector manufacturero deben potenciar el aprovechamiento de los activos totales disponibles para incrementar el volumen de ventas, generando eficiencia en la dirección del negocio.

El sector manufacturero tiene un alto nivel de representatividad en el ámbito de la producción nacional y desarrollo económico del país. Con base a lo expuesto se identifica un buen manejo de los periodos medios de cobranza y pagos. De igual manera se visualiza que en los años 2014, 2015 y 2017 en promedio se genera una mayor eficiencia en la gestión de capital de trabajo (Rotación De Cartera, Rotación De Activo Fijo, Rotación De Ventas, Periodo Medio De Cobranzas y Periodo Medio De Pago). 
Es importante recalcar que la Rotación de ventas en el análisis longitudinal representa el indicador con los resultados más bajos.

El año 2017 se conjetura como un periodo de mayor de rentabilidad del activo $(0,0498)$, en contraste con el total de los años estudiados. Los subsectores con mayor rentabilidad corresponden a la fabricación de vehículos automotores remolques y semirremolques (C29). Y reparación e instalación de maquinaria y equipo. (C33) con una media similar de 0,0406 y 0,0473 respectivamente. 


\section{REFERENCIAS}

Abad, (2011). El precio: clave de la rentabilidad. Bogotá: Planeta.

Abreu, M. (2009). El capital de trabajo de las empresas que cotizan en la bolsa mexicana de valores. Comercio Exterior, 193-2010.

Aguilar, T. S. (2017). Importancia de la administración eficiente del capital de trabajo en las Pymes. Ciencia, 30-39.

Angulo, B. (2014). Estrategias de invesión de capital de trabajo aplicadas por las micro, pequeñas y medianas empresas colombianas de comercio textil en el municipio de Maicao. Dimensión Empresarial, 69-82.

Block, D. (2013). Fundamentos de Administración Financiera. McGrawHill. : McGrawHill. .

CEPAL. (2012). Cambio estructural para la igualdad: Una visión integrada del desarrollo. . Santiago: Naciones Unidas. Obtenido de https://www.cepal.org/pses34/noticias/documentosdetrabajo/4/47424/2012-ses-34cambio_estructural.pdf

Compañias, S. d. (2020). Tabla de indicadores. Quito. Obtenido de http://reporteria.supercias.gob.ec/portal/samples/images/docs/tabla_indicadores.pdf

Contreras, D. (2015). Estructura financiera y rentabilidad: origen, teorías y definiciones. Revista Valor Contable, 35-44.

Córdova. (2007). Gerencia Financiera Empresarial. Bogotá: Ecoe Ediciones.

Córdova, M. (2013). Gestión Financiera. Bogotá: Ecoe Ediciones.

Cuenca, R. C. (2018). La gestión de capital de trabajo y su efecto en la rentabilidad de las empresas constructoras del Ecuador. X-Pedientes Económicos, 28-45.

Duran. (2011). Administración de capital de trabajo: Una herramienta financiera para la gerencia de las PYMES tradicionales Venezolanas. Visión Gerencial, 37-56.

Eslava, J. (2010). Las claves del análisis económico- financiero de la empresa. Madrid: ESIC.

Espinoza, C. (2007). Políticas de capital de trabajo y su influencia en el riezgo y la rentabilidad. Cuba: Universidad de Matanzas.

Faxas del Toro, A. (2011). El Análisis Financiero del Capital de trabajo en la empresa. México: Observatorio de la Economía Latinoamericana.

Garcìa , O., Martinez , G., \& Fernandez, G. (2018). Mercado de renta variable: Análisis de tìtulos. Madrid: Paraninfo S.A. books.google.com.ec/books?id=o_5KDwAAQBAJ\&dq=es\&hl=es\&source=gbs.

Gitman, L. J. (2012). Principios de administración financiera. México: Pearson Educacion. 
Gonzales, Q. (2012). Planeación Financiera de la Empresa. Loja.

Hamid, A. H. (2017). Relationship between working capital management and profitability. Management , 1-12.

Horma, G., \& Osorio. (2009). Análisis de mercado del sector industrias manufactureras en base a CIIU 3 bajo un enfoque de concentración económica en el período 2000-2008 en el Ecuador. Revista Politécnica, 230-243.

Jimenez, R. O. (2013). La importancia del ciclo de caja y cálculo de capital de trabajo. Clio America, 4863.

Miller. (2014). Administración de capital de trabajo, liquidez y rentabilidad en el sector textil de Cúcúta, periodo 2008 - 2011. Respuestas , 86-98.

Monsreal, J., Ku-Tuz, J., Serralta, L., Sánchez, M. (2020). Statistical comparison of the external validity (positive predictive value and negative predictive value) of three anthropometric indices used in the diagnosis of pathological nutritional situations overweight and obesity, South Florida Journal of Development, Miami, v.2, n.1, p.685-700. DOI: 10.46932/sfjdv2n1-050.

Morlás, C. (18 de 07 de 2015). ¿Continuará siendo rentable el negocio bancario privado en el Ecuador? Obtenido de ¿Continuará siendo rentable el negocio bancario privado en el Ecuador?: https://ideas.repec.org/a/erv/observ/y2015i20718.html

Navarro, C. L. (2018). Perspectivas de la administración financiera de capital de trabajo como instrumento necesario para la evolución de las PyMes. Espacios, 14-24.

Porlles, Q. C. (2013). Pronostico Financiero: Metodos rapidos de estimulación del fondo de maniobra o capital de trabajo. Red de revistas científicas para América Latina. Industrial Data, 29-36.

Redondo, R., \& Arias, J. (2013). Teoría de la finanaciación. Madrid: Universidad Nacional de Educación a distancia.

Rizzo. (2007). El capital de trabajo neto y el valor de las empresas: La importancia de la recomposición del capital de trabajo neto en las empresas que atraviesan o han atravesado crísis financieras. Escuela de Administración de negocios, 103-121.

Ross, W. J. (2012). Finanzas Corporativas. México: McGraw-Hill.

Rotstein, F. E. (2004). La administración eficiente del capital como instrumento para detectar y prevenir fracasos financieros. Escritos Contables, 63-84.

Saucedo, H. (2019). Auditoría Financiera de Capital de Trabajo en Pymes. México: Instituto México de Contadores Públicos.

Soriano, C. (2010). Introducción a la contabilidad y las finanzas: Incluye ejemplos y. Barcelona: Profit Editorial.

Superintendencia de Compañías, V. y. (2018). Panorama de la Industria Manufacturera en el Ecuador 2013 - 2017. Quito: DIRECCIÓN NACIONAL DE INVESTIGACIÓN Y ESTUDIOS. Obtenido de 
https://investigacionyestudios.supercias.gob.ec/wp-content/uploads/2018/09/Panorama-de-la-IndustriaManufacturera-en-el-Ecuador-2013-2017.pdf

Superintendencia de Compañías, V. y. (2020). La eficiencia en las empresas manufactureras del Ecuador 2013 - 2018. Quito: DIRECCIÓN NACIONAL DE INVESTIGACIÓN Y ESTUDIOS.

Van Horne, J., \& Wachowicz, J. (2010). Fundamento de la administración financiera. México: Prentice Hall.

Vera, M. M. (2013). Acceso a la información Pimes. Innovar, 149-160.

Zamora. (2008). Rentabilidad y Ventaja Comparativa: Un Análisis de los Sistemas de Producción de Guayaba en el Estado de Michoacán. México: Universidad Michoacana de San Nicolás de Hidalgo. 